\title{
Is an HIV Vaccine Possible?
}

\author{
Nancy A. Wilson ${ }^{1,2}$ and David I. Watkins ${ }^{1,2}$ \\ ${ }^{1}$ Department of Pathology and Laboratory Medicine, University of Wisconsin-Madison; ${ }^{2}$ Wisconsin National Primate Research Center, University \\ of Wisconsin-Madison; Madison, Wisconsin, USA
}

\begin{abstract}
The road to the discovery of a vaccine for HIV has been arduous and will continue to be difficult over the ensuing twenty years. Most vaccines are developed by inducing neutralizing antibodies against the target pathogen or by using attenuated strains of the particular pathogen to engender a variety of protective immune responses. Unfortunately, simple methods of generating anti-HIV antibodies have already failed in a phase III clinical trial. While attenuated SIV variants work well against homologous challenges in non-human primates, the potential for reversion to a more pathogenic virus and recombination with challenge viruses will preclude the use of attenuated HIV in the field. It has been exceedingly frustrating to vaccinate for HIV-specific neutralizing antibodies given the enormous diversity of the Envelope (Env) glycoprotein and its well-developed glycan shield. However, there are several antibodies that will neutralize many different strains of HIV and inducing these types of antibodies in vaccinees remains the goal of a vigorous effort to develop a vaccine for HIV based on neutralizing antibodies. Given the difficulty in generating broadly reactive neutralizing antibodies, the HIV vaccine field has turned its attention to inducing $\mathrm{T}$ cell responses against the virus using a variety of vectors. Unfortunately, the results from Merck's phase IIb STEP trial proved to be disappointing. Vaccinees received Adenovirus type 5 (Ad5) expressing Gag, Pol, and Nef of HIV. This vaccine regimen failed to either prevent infection or reduce the level of HIV replication after challenge. These results mirrored those in non-human primate testing of Ad5 using rigorous SIV challenge models. This review will focus on recent developments in HIV vaccine development. We will deal largely with attempts to develop a T cell-based vaccine using the non-human primate SIV challenge model.
\end{abstract}

Key-Words: HIV, vaccine, challenges.

SIV challenge of non-human primates is perhaps the best way to test HIV vaccine concepts [1-6]. SIV and HIV share similar genomic organizations [7]. Similar to HIV, SIV uses CCR5 to infect activated CD4 cells [8-10]. And SIV, like HIV, depletes activated CD4 cells from the gut immediately after infection and causes a slow decline in total CD4 cells over time, eventually leading to opportunistic infections and subsequently death [11, 12]. SIV challenge of non-human primates thus recapitulates many of the essential elements of HIV infection. Unfortunately, most SIV challenges of Indian rhesus macaques take place with viruses that have sequence similarity to the vaccine SIV sequences [6, 13-17]. These homologous challenges do not replicate the kinds of HIV exposures that human vaccinees will face in the field. We will first discuss homologous challenges and then more recent results from heterologous challenges.

\section{Challenge Virus}

There are two main SIV challenges viruses utilized and they each have their own pros and cons. Most challenges to date have been performed using the closely related swarm virus SIVmac251 or the pathogenic clone SIVmac239 [4-6, 1720]. Several challenges have also been performed using SIVsmE660 [20-22], which has sequence dissimilarities from

Received on 3 July 2009; revised 31 July 2009.

Address for correspondence: Dr. David I Watkins or Nancy A. Wilson. University of Wisconsin - Madison. AIDS Vaccine Development Laboratory. 555 Science Drive. Madison, WI 53711. (608) 263-5953 (phone). (608) 265-8084 (FAX). E-mail: nwilson@primate.wisc.edu.

The Brazilian Journal of Infectious Diseases

2009;13(4):304-310. (C) 2009 by The Brazilian Journal of Infectious Diseases and Contexto Publishing. All rights reserved.
SIVmac239 and SIVmac251 making it a bona fide heterologous challenge. However, SIVmac251 and 239 in particular have high viral set points of between 100,000 and 500,000, quite different from the viral set points of 30,000 seen in HIV-infected humans. SIVsmE660 on the other hand, has virus loads that are considerably lower in the chronic phase. Because it is a swarm virus, considerable animal-to-animal variability has also been observed with after mucosal challenge with this virus. This animal-to-animal variability can also be stock dependent. That is, different laboratories can have different stocks of virus, which can have different outcomes after challenge.

\section{Homologous Challenges}

It is now clear that vaccine-induced $\mathrm{T}$ cells can reduce virus replication after challenge with the highly pathogenic SIVmac239 [23]. Vaccination using three DNA primes and a single Ad5 boost expressing Gag, Tat, Nef, and Rev of SIVmac239 reduced peak viremia from 50 million to 4 million in Indian rhesus macaques repeatedly challenged mucosally with the highly pathogenic SIVmac239 clone $(p=0.007)$. Furthermore, virus replication in the chronic phase was only 5,300 in the seven vaccinees compared to a set point of 150,000 in six control animals $(p=0.0192)$ (Figure 1$)$. Since this vaccine regimen did not include Env, no Env- specific antibodies were engendered, and we can conclude that $\mathrm{T}$ cells raised by the vaccine controlled both peak and set point plasma viremia in this model utilizing a low dose repeated rectal SIVmac239 challenge.

These results were confirmed recently using an Ad5/Ad26 prime/boost utilizing SIVmac239 Gag sequences in Indian rhesus macaques [24]. Here vaccinated animals were challenged intravenously with SIVmac251 and peak virus load 
was reduced by a factor of $1.4 \operatorname{logs}\left(29.5 \times 10^{6}\right.$ to $1.1 \times 10^{6}, \mathrm{p}=$ 0.002 ) and virus load at the set point in the vaccinees was 2.44 logs lower $\left(3.7 \times 10^{3}\right.$ compared to $\left.1.02 \times 10^{6}\right)$ than control animals in this study $(\mathrm{p}=0.01)$. More recently, Louis Picker's group has recently shown that four animals vaccinated with a CMV vector expressing Gag, Tat, Rev, Nef, and Env controlled replication of the highly pathogenic SIVmac239 virus to small blips of viremia during the acute phase [25]. Two of these animals had only one or two positive readings of less than 100 copies/mL suggesting that the vaccine induced by the $\mathrm{CMV}$-vectored inserts can achieve a remarkable level of control over acute phase replication. While Env was included in this particular vaccine regimen, no Env-specific neutralizing antibodies were present at time of challenge. Thus, vaccines designed to induce $T$ cells can control replication of SIV without neutralizing antibodies.

There are, however, a number of caveats to these studies. The first is what sort of damage was inflicted by $10^{6}$ copies/ $\mathrm{mL}$ during the acute phase in the first two studies described above? It's likely that the vaccinees lost the majority of their CD4 memory cells during acute phase virus replication and this will likely compromise the immune response to both SIV and a variety of other opportunistic pathogens later in the course of SIV infection. The other and perhaps more serious issue is that the vaccine and the challenge viruses were exactly matched (homologous challenge) in all three described studies. This is unlikely to happen with HIV vaccinees challenged in the field.

The goal of an HIV vaccine based on $\mathrm{T}$ cell responses should, therefore, be to limit virus replication during the acute phase and exert complete control in the chronic phase against a heterologous virus. Indeed, replicating the sort of encouraging data generated by the Picker Laboratory [25] with a heterologous challenge should be the goal of HIV vaccine development. That is two or three positive viral readings during the acute phase and then no evidence of virus replication in the chronic phase after a rigorous heterologous challenge (Figure 2).

\section{Heterologous Challenge}

We recently conducted an experiment exploring whether $\mathrm{T}$ cell responses against a variety of different epitopes could control replication of SIV after a heterologous challenge [26]. We vaccinated eight animals with three DNA primes and a single non-replicating Ad5 boost with both the DNA and Ad5 vectors expressing all of the proteins in SIV proteome except for Env. We did not include Env because we were trying to address the role of vaccine-induced cellular $T$ cell response in control of virus replication. Additionally, we excluded all animals expressing rhesus macaque MHC class I alleles that have previously been associated with control of virus replication, namely $М а т и-A * 01$, Мати- ${ }^{*} 08$, and $М а т и-$ $B * 17[13,16,27-29]$. All animals were $M a m u-A * 02$ positive so that we could follow antigen-specific $\mathrm{T}$ cell responses directed against epitopes bound by this particular MHC class I molecule [30]. Our vaccine induced high frequency $\mathrm{T}$ cell responses against the majority of the proteins in the vaccine. Using IFN $\gamma$ Elispot, we detected an average of 12,000 SFC $/ 10^{6}$ PBMC in our eight vaccinees. Gag and Vif induced the highest frequency $\mathrm{T}$ cell responses. The vaccinees recognized an average of twenty different regions (epitopes) in the virus with Gag and Vif again being the most heavily targeted. We then challenged these animals with the heterologous swarm virus SIVsmE660. This virus differed from the vaccine strain by as much as one in four amino acids in Tat and one in twenty amino acids in p15 and p27 of Gag. This challenge mimicked the kind of challenge that a vaccinee might expect when the HIV exposure is of the same clade as the vaccine [31]. That is, a within-clade challenge. We also titrated our challenge virus so that one to three variants crossed the mucosal membrane. Again, we were trying to mimic the situation in humans where more recently it has been shown that in the majority of mucosal infections, only one to three different clones of HIV replicate in the acute phase. At 61 weeks after the first DNA vaccination, we challenged our animals with repeated mucosal exposures to 800 TCID $_{50}(1.2 \mathrm{x}$ $10^{7}$ viral copies/mL) of the heterologous swarm virus SIVsmE660. We carried out five challenges at this dose and if animals remained uninfected, we followed this with six doses of 4,000 $\mathrm{TCID}_{50}\left(6 \times 10^{7}\right.$ viral copies $\left./ \mathrm{mL}\right)$.

Our vaccine did not offer protection against acquisition of SIV infection. It took an average of four challenges to infect our eight vaccinees and four challenges to infect our control animals. This suggests that a $\mathrm{T}$ cell-based vaccine may not offer protection against acquisition of SIV infection.

We infected all eight animals in our control group. Two of eight animals have already succumbed to AIDS-associated opportunistic infections, each with virus loads above a million copies/mL in the chronic phase. One of the animals has an undetectable virus load at 45 weeks post infection and three additional animals have virus loads between 100 and 1,000 copies/mL at 40 weeks post infection. The other two animals have virus loads of close to a million copies at 40 weeks post infection. Using a low dose challenge model, we have now infected all eight of our naïve control animals recapitulating HIV infection of humans.

The results from the challenge of the vaccinees were quite unexpected. At 40 weeks post infection, we could not detect virus in six of our eight vaccinees. Most importantly six of the eight vaccinees had acute peaks of less than one million copies/mL. Thus this vaccine, while it did not prevent infection, controlled acute phase virus replication to less than 10,000 copies/mL and exerted a measure of control in the chronic phase with vaccinees averaging approximately 210 copies/ $\mathrm{mL}$ (Figure 3). The naïve unvaccinated animals in this study had peak viremias of over a million copies/mL with a chronic virus level of approximately 80,000 copies/mL, similar to the chronic phase levels of virus replication in HIV-infected humans. Thus, this vaccine, for the first time, not only controlled chronic phase virus replication but also succeeded 
Figure 1. Control of chronic phase viral replication achieved using a vaccine homologous to the challenge virus. We vaccinated eight rhesus macaques expressing Мати- $A * 01$ with DNA (three primes) and Ad5 (single boost) encoding Gag, Tat, Rev and Nef from SIVmac239. We then challenged the eight vaccinees (black closed triangles, solid line) and eight naïve animals (open triangles and dotted line) with SIVmac239 using a low dose intrarectal challenge protocol. Vaccinees experienced a log drop in peak (acute) viremia and a 1.5 log reduction in chronic viremia, both of which were statistically significant.

\section{Gag, Tat, Rev, Nef}

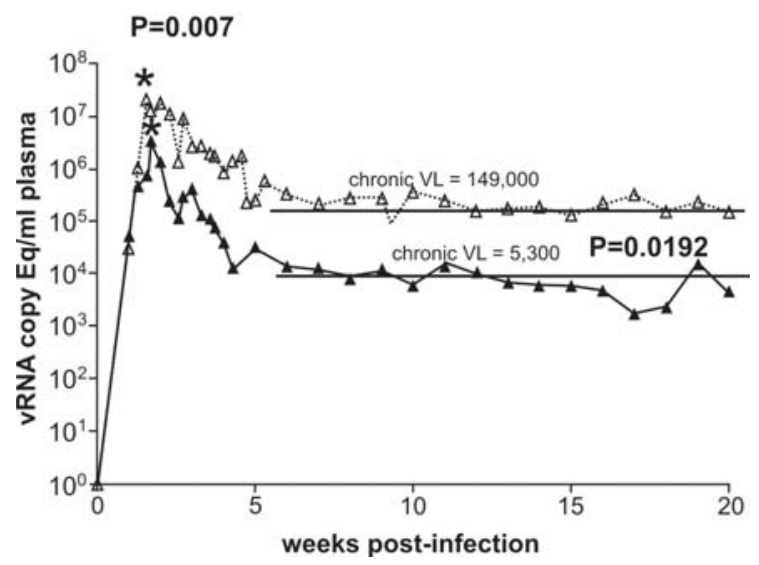

Figure 2. An ideal T cell based vaccine would result in only limited viral replication during the acute phase, and viral replication would be undetectable in the chronic phase. The dashed line represents HIV viral replication in naïve persons, and the solid line shows viral replication in protected vaccinees. Limiting viral replication in the acute phase would preserve the crucial CD4+ T cell subset, as these cells would be protected from infection and destruction in the acute phase. Transmission and generation of new mutants throughout the infection would be also greatly reduced due to overall lower viral replication.

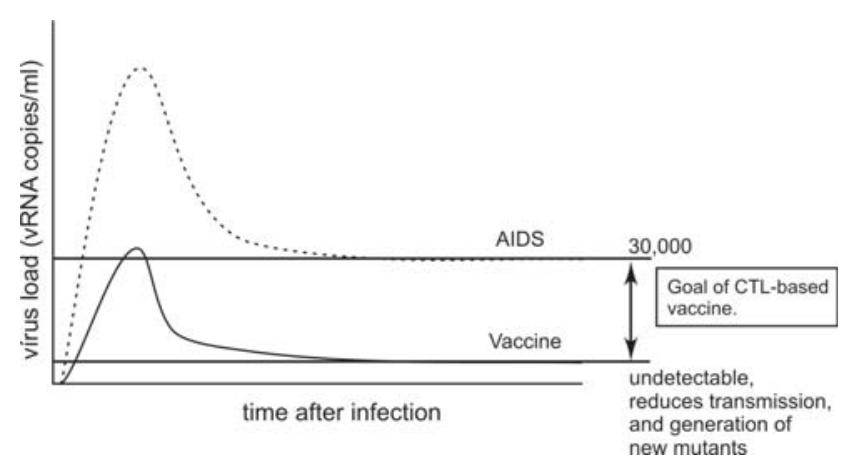

Figure 3. Control of both acute and chronic phase viral replication was achieved using a vaccine encoding all SIV proteins except Env, and a homologous mucosal challenge. We vaccinated eight rhesus macaques expressing Мamu$A * 02$ (we excluded animals that expressed Mamu- $A^{*} 01$, Mamu- $B^{*} 08$ or $M a m u-B^{*} 17$ ) with DNA (three primes) and Ad5 (single boost) encoding Gat, Tat, Rev, Nef, Pol, Vif, Vpr and Vpx from SIVmac239. We then challenged the eight vaccinees (black closed diamonds, solid line) and eight naïve animals (open diamonds, dotted line), that also expressed Мати- $A^{*} 02$ (we excluded animals that expressed Mamu$A * 01$, Mamu-B*08 or Mamu-B*17), mucosally with up to five low dose challenges and up to six high dose challenges of the heterologous swarm virus SIVsmE660. Vaccinees controlled viral replication in both the acute (peak) phase and chronic phase.

\section{Gag, Tat, Rev, Nef, Pol, Vif, Vpr, Vpx}

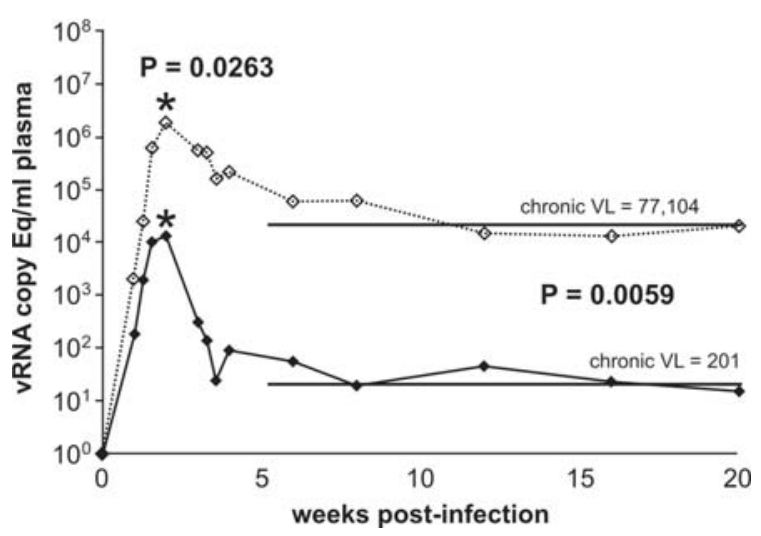

in controlling acute phase virus replication. This control of acute phase viral replication may have reduced the damage to the all-important CD4 memory cell compartment, hopefully allowing a robust immune response to the infecting virus.

The vaccinees mounted high frequency anamnestic vaccine-induced $\mathrm{T}$ cell responses during the acute phase of challenge. One of the animals (r02103) that had six positive acute phase virus loads, all less than 300 copies/mL, mounted high frequency anamnestic $\mathrm{T}$ cell responses against several Mamu-A*02 bound peptides. For example, the T cell response against the Mamu-A*02-restricted GagGY9 epitope was 50 $\mathrm{SFC} / 10^{6} \mathrm{PBMC}$ on day of challenge rising to $2500 \mathrm{SFC} / 10^{6}$ $\mathrm{PBMC}$ at three weeks post infection during the acute phase of virus replication. Indeed, several of the $\mathrm{T}$ cell responses raised by the vaccine were expanded anamnestically (Figure $4 \mathrm{~A}, \mathrm{~B})$. Given the heterologous nature of the challenge, only approximately half of the $T$ cell responses raised by the vaccine were expanded post challenge. Surprisingly, we observed massive expansions of CD4 T cells induced by vaccination post challenge (Figure 4). Indeed, the majority of the T cells, both in terms of frequency and in terms of epitopes recognized 
Figure 4. Broad vaccine induced anamnestic responses are present in animals that had limited viral replication at peak, and continue to control viremia to very low or undetectable levels in the chronic phase. Each panel shows the frequency of anamnestic responses at 2-3 weeks post challenge as measured by Elispot using pools of peptides (indicated on the $\mathrm{x}$ axis). Black bars represent responses seen in the entire PBMC population (panels A, C, E, G and I) whereas the responses represented by white bars are those observed in PBMC that have been depleted of CD8+ cells, which thereby represent CD4+ T cell responses (panels B, D, F, H, J). Peak and chronic viremia for each animal are shown in boxes. Since the entire PBMC population contains both CD4+ and CD8+ T cells, it is possible that these responses are present only in CD4+ T cells, but are observed in both populations. Responses that were found in both whole PBMC, as well as in CD8-depleted PBMC (CD4s) are indicated with a star (*) above the bar.

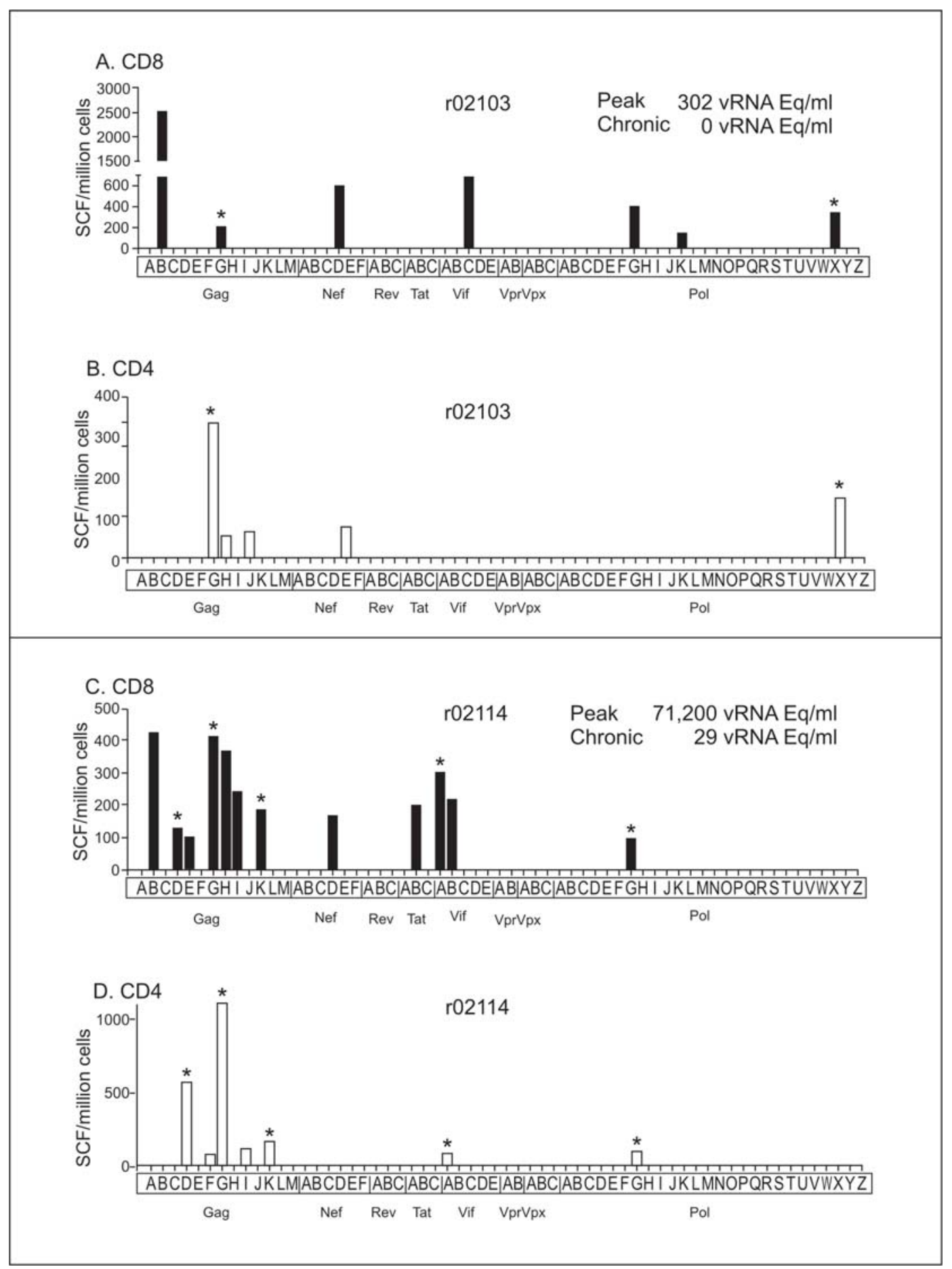

www.bjid.com.br 
after challenge, came from the CD4 compartment. Is it possible that it was the vaccine-induced CD4 cells that were responsible for the success of this $\mathrm{T}$ cell-based vaccine? This is an intriguing hypothesis that probably should be explored in subsequent experiments.

\section{Speculations}

The replication-deficient Ad5-vectored STEP trial induced CD8 responses against very few epitopes in the HIV Gag, Pol, and Nef inserts [32,33]. Future analyses hopefully will determine why $\mathrm{T}$ cell responses were raised against such a limited number of $\mathrm{T}$ cell epitopes. It is possible that preexisting antibody and $\mathrm{T}$ cell responses against the vector itself affected the $\mathrm{T}$ cell response to the inserts. We should perhaps explore vectors that are smaller and thus give the immune system a restricted choice of immunodominant epitopes in the vector itself. These immunodominant vector-specific T cell responses may drown out the $\mathrm{T}$ cell responses to the eptiopes encoded for by the HIV or SIV inserts. Additionally, it may be that we need to deliver these large SIV or HIV viral open reading frames in small segments to overcome immunodominance issues. By that, we mean that the dominant $\mathrm{T}$ cell response to an epitope either in the vector or the insert may preclude the development of CD8 T cell responses to subdominant epitopes that may be efficacious in the face of viral challenge.

It is possible to make a T cell-based vaccine. Of course it would be ideal to induce neutralizing antibodies. A combination of both vaccine-induced antibodies and $\mathrm{T}$ cells would be optimal. But unfortunately we don't have any candidate antibody-based vaccines yet. We know that attenuated SIV vaccines likely work because of MHC class I-CD8 interactions [34]. Recently, it has been shown that several vaccine regimens in which Env has been purposefully excluded can control replication in both the peak and chronic phase after both homologous and heterologous challenge [23-26]. Finally, the hallmark of rare individuals who control replication of SIV and HIV replication is the expression of certain key MHC class I alleles; HLA-B*57 and -B*27 in humans and Mamu-B*08 and $-\mathrm{B}^{*} 17$ in monkeys [27-29, 35-45]. Cellular immune responses can, therefore, control replication of both SIV and HIV without the help of neutralizing antibodies. Thus, we should devote considerable effort to try to develop vaccine regimens designed to induce CD8 and CD4 T cell responses. These vaccines should be tested with heterologous SIV challenges in non-human primates and if they show measures of control, should be advanced into phase IIb clinical trials. Phase I clinical trials should be undertaken at the beginning of these vector explorations because different vectors may have different effects in humans and monkeys.

\section{Conclusion}

We have shown, for the first time, that it is possible to control both the acute and chronic phase of viral replication using vaccine-induced $\mathrm{T}$ cell responses. This control was achieved in the complete absence of antibodies against the
Env glycoprotein. The vaccinees averaged 32,000 copies/mL at peak where the controls had an average of 2.5 million copies/ $\mathrm{mL}$ at peak. Virus was undetectable in six of eight of the vaccinees by 40 weeks post challenge. There are, however, a number of caveats with regard to this study. This SIVsmE660 challenge is a novel challenge and an important experiment would be to see whether the Ad5 Gag/Pol/Nef vaccine regimen used in the STEP protocol could control replication of SIVsmE660 delivered by repetitive low doses across the mucosa. Additionally, it would be interesting to see whether our best vaccine, attenuated SIVmac239DNef, can control replication of SIVsmE660 after repeated mucosal challenge. Both of these experiments are now underway. Finally, a key issue would be how long this measure of control will last.

It has been difficult to find a vaccine for HIV. Classical methods of inducing antibodies and using live attenuated viruses have failed or are likely to be too dangerous to use in human populations $[34,46]$. It has been impossible to date to induce broadly reactive neutralizing antibodies, the holy grail of HIV vaccine development. The field has therefore turned to a novel, and as yet untested, vaccine approach to induce $\mathrm{T}$ cell responses against SIV or HIV. This movement suffered a minor setback when the STEP trial using Merck's Ad5-vectored Gag, Pol, Nef failed last year [32, 33]. However, this result should not dampen our enthusiasm for making $\mathrm{T}$ cell-based vaccines against HIV. This Merck vaccine induced $\mathrm{T}$ cell responses against only three HIV epitopes. Such a limited number of epitopes was unlikely to be effective against a heterologous HIV challenge. Several recent studies have shown that vaccine-induced $\mathrm{T}$ cell responses can control replication of both the acute phase and chronic phase of SIV replication using both homologous and heterologous challenges [23-26]. If anything, we need to redouble our efforts to test as many different vectors as we can against this variable pathogen. Testing the ability of these vectors to induce T cell responses against SIV proteins in non-human primates by rigorous SIV heterologous challenges is the only way we can prioritize our vaccine candidates for moving into phase IIb clinical trials.

\section{Acknowledgements}

We are grateful to the staff at the Wisconsin National Primate Research Center (WNPRC) for the expert care of animals used in this study. We thank Jason Reed for technical help. We thank the MHC Typing Core at WNPRC for PCRSSP typing of these animals. We thank the Virology Core at WNPRC for viral load determinations. We thank the Immunology Core at WNPRC for assistance with blood processing, Elispot assays and maintenance of the flow cytometry facility. D.I.W. and his laboratory are supported by the U.S. National Institutes of Health (grants R01 AI049120, R37 AI052056, R01AI076114, R24 RR015371, R24 RR016038, and R21 AI077472; contract HHSN266200400088C). This publication was made possible in part by P51 RR000167 from the Wisconsin National Primate Research Center. This research 
was conducted at a facility constructed with support from the Research Facilities Improvement Program grants RR15459-01 and RR020141-01. The Delta Nef studies were supported by funds from the International AIDS Vaccine Initiative.

\section{References}

1. Baba T.W., Liska V., Hofmann-Lehmann R. et al. Human neutralizing monoclonal antibodies of the IgG1 subtype protect against mucosal simian-human immunodeficiency virus infection. Nat Med 2000; 6:200-206.

2. Mascola J.R., Stiegler G., VanCott T.C. et al. Protection of macaques against vaginal transmission of a pathogenic HIV-1/SIV chimeric virus by passive infusion of neutralizing antibodies. Nat Med 2000; 6:207-210.

3. Parren P.W., Marx P.A., Hessell A.J. et al. Antibody protects macaques against vaginal challenge with a pathogenic R5 simian/ human immunodeficiency virus at serum levels giving complete neutralization in vitro. J Virol 2001; 75:8340-8347.

4. Allen T.M., Jing P., Calore B. et al. Effects of cytotoxic T lymphocytes (CTL) directed against a single simian immunodeficiency virus (SIV) Gag CTL epitope on the course of SIVmac239 infection. J Virol 2002; 76:10507-10511.

5. Allen T.M., Mortara L., Mothe B.R. et al. Tat-vaccinated macaques do not control simian immunodeficiency virus SIVmac239 replication. J Virol 2002; 76:4108-4112.

6. Vogel T.U., Reynolds M.R., Fuller D.H. et al. Multispecific vaccineinduced mucosal cytotoxic $\mathrm{T}$ lymphocytes reduce acute-phase viral replication but fail in long-term control of simian immunodeficiency virus SIVmac239. J Virol 2003; 77:1334813360.

7. Bontrop R.E. Non-human primates: essential partners in biomedical research. Immunol Rev 2001; 183:5-9.

8. Kirchhoff F., Pohlmann S., Hamacher M. et al. Simian immunodeficiency virus variants with differential T-cell and macrophage tropism use CCR5 and an unidentified cofactor expressed in CEMx174 cells for efficient entry. J Virol 1997; 71:6509-6516.

9. Chen Z., Zhou P., Ho D.D., Landau N.R., and Marx P.A. Genetically divergent strains of simian immunodeficiency virus use CCR5 as a coreceptor for entry. J Virol 1997; 71:2705-2714.

10. Chen Z., Gettie A., Ho D.D., and Marx P.A. Primary SIVsm isolates use the CCR5 coreceptor from sooty mangabeys naturally infected in west Africa: a comparison of coreceptor usage of primary SIVsm, HIV-2, and SIVmac. Virology 1998; 246:113124.

11. Mattapallil J.J., Douek D.C., Hill B. et al. Massive infection and loss of memory CD4+ $\mathrm{T}$ cells in multiple tissues during acute SIV infection. Nature 2005; 434:1093-1097.

12. Li Q., Duan L., Estes J.D. et al. Peak SIV replication in resting memory CD4+ T cells depletes gut lamina propria CD4+ T cells. Nature 2005; 434:1148-1152.

13. Casimiro D.R., Wang F., Schleif W.A. et al. Attenuation of simian immunodeficiency virus SIVmac239 infection by prophylactic immunization with dna and recombinant adenoviral vaccine vectors expressing Gag. J Virol 2005; 79:15547-15555.

14. Engram J.C., Dunham R.M., Makedonas G. et al. Vaccine-induced, Simian Immunodeficiency Virus-specific CD8+ T cells reduce virus replication but do not protect from Simian Immunodeficiency Virus disease progression. J Immunol 2009; 183:706-717.

15. Horton H., Vogel T.U., Carter D.K. et al. Immunization of rhesus macaques with a DNA prime/modified vaccinia virus Ankara boost regimen induces broad simian immunodeficiency virus (SIV)-specific T-cell responses and reduces initial viral replication but does not prevent disease progression following challenge with pathogenic SIVmac239. J Virol 2002; 76:7187-7202.
16. McDermott A.B., O’Connor D.H., Fuenger S. et al. Cytotoxic Tlymphocyte escape does not always explain the transient control of simian immunodeficiency virus SIVmac239 viremia in adenovirus-boosted and DNA-primed Mamu-A*01-positive rhesus macaques. J Virol 2005; 79:15556-15566.

17. Vaccari M., Mattapallil J., Song K. et al. Reduced protection from simian immunodeficiency virus SIVmac251 infection afforded by memory CD8+ $\mathrm{T}$ cells induced by vaccination during CD4+ T-cell deficiency. J Virol 2008; 82:9629-9638.

18. Barouch D.H., Liu J., Lynch D.M. et al. Protective Efficacy of a Single Immunization of a Chimeric Adenovirus Vector-Based Vaccine Against SIV Challenge in Rhesus Monkeys. J Virol 2009;

19. Demberg T., Boyer J.D., Malkevich N. et al. Sequential priming with simian immunodeficiency virus (SIV) DNA vaccines, with or without encoded cytokines, and a replicating adenovirus-SIV recombinant followed by protein boosting does not control a pathogenic SIVmac251 mucosal challenge. J Virol 2008; 82:10911-10921.

20. Yeh W.W., Jaru-Ampornpan P., Nevidomskyte D. et al. Partial protection of Simian immunodeficiency virus (SIV)-infected rhesus monkeys against superinfection with a heterologous SIV isolate. J Virol 2009; 83:2686-2696.

21. Abdel-Motal U.M., Gillis J., Manson K. et al. Kinetics of expansion of SIV Gag-specific CD8+ T lymphocytes following challenge of vaccinated macaques. Virology 2005; 333:226-238.

22. Brown C.R., Czapiga M., Kabat J. et al. Unique pathology in simian immunodeficiency virus-infected rapid progressor macaques is consistent with a pathogenesis distinct from that of classical AIDS. J Virol 2007; 81:5594-5606.

23. Wilson N.A., Reed J., Napoe G.S. et al. Vaccine-induced cellular immune responses reduce plasma viral concentrations after repeated low-dose challenge with pathogenic simian immunodeficiency virus SIVmac239. J Virol 2006; 80:5875-5885.

24. Liu J., O’Brien K.L., Lynch D.M. et al. Immune control of an SIV challenge by a T-cell-based vaccine in rhesus monkeys. Nature 2009; 457:87-91.

25. Hansen S.G., Vieville C., Whizin N. et al. Effector memory T cell responses are associated with protection of rhesus monkeys from mucosal simian immunodeficiency virus challenge. Nat Med 2009; 15:293-299.

26. Wilson N.A., Keele B.F., Reed J.S. et al. Vaccine-induced cellular responses control simian immunodeficiency virus replication after heterologous challenge. J Virol 2009; 83:6508-6521.

27. Loffredo J.T., Maxwell J., Qi Y. et al. Mamu-B*08-positive macaques control simian immunodeficiency virus replication. J Virol 2007; 81:8827-8832.

28. Maness N.J., Yant L.J., Chung C. et al. Comprehensive immunological evaluation reveals surprisingly few differences between elite controller and progressor Mamu-B*17-positive Simian immunodeficiency virus-infected rhesus macaques. J Virol 2008; 82:5245-5254.

29. Yant L.J., Friedrich T.C., Johnson R.C. et al. The high-frequency major histocompatibility complex class I allele Mamu-B*17 is associated with control of simian immunodeficiency virus SIVmac239 replication. J Virol 2006; 80:5074-5077.

30. Loffredo J.T., Sidney J., Wojewoda C. et al. Identification of seventeen new simian immunodeficiency virus-derived CD8+ T cell epitopes restricted by the high frequency molecule, Mamu- $\mathrm{A}^{*} 02$, and potential escape from CTL recognition. J Immunol 2004; 173:5064-5076.

31. Gaschen B., Taylor J., Yusim K. et al. Diversity considerations in HIV-1 vaccine selection. Science 2002; 296:2354-2360.

32. Buchbinder S.P., Mehrotra D.V., Duerr A. et al. Efficacy assessment of a cell-mediated immunity HIV-1 vaccine (the Step Study): a double-blind, randomised, placebo-controlled, test-of-concept trial. Lancet 2008; 372:1881-1893.

33. McElrath M.J., De Rosa S.C., Moodie Z. et al. HIV-1 vaccineinduced immunity in the test-of-concept Step Study: a casecohort analysis. Lancet 2008; 372:1894-1905. 
34. Reynolds M.R., Weiler A.M., Weisgrau K.L. et al. Macaques vaccinated with live-attenuated SIV control replication of heterologous virus. J Exp Med 2008; 205:2537-2550.

35. Loffredo J.T., Sidney J., Bean A.T. et al. Two MHC class I molecules associated with elite control of immunodeficiency virus replication, Mamu-B*08 and HLA-B*2705, bind peptides with sequence similarity. J Immunol 2009; 182:7763-7775.

36. Carrington M., and O'Brien S.J. The influence of HLA genotype on AIDS. Annu Rev Med 2003; 54:535-551.

37. Gao X., Bashirova A., Iversen A.K. et al. AIDS restriction HLA allotypes target distinct intervals of HIV-1 pathogenesis. Nat Med 2005; 11:1290-1292.

38. Altfeld M., Kalife E.T., Qi Y. et al. HLA Alleles Associated with Delayed Progression to AIDS Contribute Strongly to the Initial CD8(+) T Cell Response against HIV-1. PLoS Med 2006; 3:e403.

39. Hendel H., Caillat-Zucman S., Lebuanec H. et al. New class I and II HLA alleles strongly associated with opposite patterns of progression to AIDS. J Immunol 1999; 162:6942-6946.

40. Goulder P.J., Phillips R.E., Colbert R.A. et al. Late escape from an immunodominant cytotoxic T-lymphocyte response associated with progression to AIDS. Nat Med 1997; 3:212-217.
41. Kaslow R.A., Rivers C., Tang J. et al. Polymorphisms in HLA class I genes associated with both favorable prognosis of human immunodeficiency virus (HIV) type 1 infection and positive cytotoxic T-lymphocyte responses to ALVAC-HIV recombinant canarypox vaccines. J Virol 2001; 75:8681-8689.

42. Kawashima Y., Pfafferott K., Frater J. et al. Adaptation of HIV-1 to human leukocyte antigen class I. Nature 2009; 458:641-645.

43. Gillespie G.M., Kaul R., Dong T. et al. Cross-reactive cytotoxic T lymphocytes against a HIV-1 p24 epitope in slow progressors with B*57. AIDS 2002; 16:961-972.

44. Goulder P.J., Tang Y., Pelton S.I., and Walker B.D. HLA-B57restricted cytotoxic T-lymphocyte activity in a single infected subject toward two optimal epitopes, one of which is entirely contained within the other. J Virol 2000; 74:5291-5299.

45. Migueles S.A., Sabbaghian M.S., Shupert W.L. et al. HLA B*5701 is highly associated with restriction of virus replication in a subgroup of HIV-infected long term nonprogressors. Proc Natl Acad Sci U S A 2000; 97:2709-2714.

46. Kim E.Y., Busch M., Abel K. et al. Retroviral recombination in vivo: viral replication patterns and genetic structure of simian immunodeficiency virus (SIV) populations in rhesus macaques after simultaneous or sequential intravaginal inoculation with SIVmac239Deltavpx/Deltavpr and SIVmac239Deltanef. J Virol 2005; 79:4886-4895. 